\title{
THE INFLUENCE OF MUSCLE FORCES ON MUSCULOSKELETAL SYSTEM LOADS DURING LIFTING - PILOT STUDY
}

\author{
A. Bieniek ${ }^{*}$, R. Michnik ${ }^{* *}, P$. Wodarski ${ }^{* * *}$, M. Chrzan ${ }^{\dagger}$, J. Jurkojé $^{\ddagger}$
}

\begin{abstract}
Present study tries to determine the effect of considering the muscles on the results of loads on the musculoskeletal system, for two basic techniques of lifting the object: stoop and squat. The trials were carried out for 5 selected positions of each technique, using AnyBody software and a verified model. Reactions in the intervertebral disc L5S1 and knee joint as well as the sum of squares of muscle forces were investigated. The obtained results confirm that the results of loads on the musculoskeletal system are an order of magnitude larger for models considering muscle forces. These studies are the introduction to a broader analysis of the problem of the influence of muscle forces on the loads of individual joints during lifting.
\end{abstract}

Keywords: human lifting, inverse dynamics, digital human modelling, muscle force, optimization

\section{Introduction}

Lifting objects is widely analysed by researchers. The main object of the analyses are loads in the lumbar spine, which are one of the basic risk factors of low back pain (Van Dieën, Hoozemans and Toussaint, 1999). In the ergonomic context, the most important thing is to define lifting techniques that minimize especially: loads on musculoskeletal system and muscle recruitment, which provides to muscle fatigue. The effect of redirecting attention to activities unrelated to the lifting activity on its kinematics and skeletal system loads were determined (Katsuhira et al., 2013). The influence of the employee's experience on kinematics of lifting and skeletal system loads was determined (Plamondon et al., 2014). The influence of the number of people carrying the object on the musculoskeletal system loads was determined (Visser et al., 2015). There were also attempts to predict and optimize the lifting path (Chang et al., 2001). The influence of the applied methodology of modelling the loads in musculoskeletal system on the obtained results were also examined (Rajaee et al., 2015). Finally the review study of literature about lifting techniques were made (Van Dieën, Hoozemans and Toussaint, 1999). Current methods of analysis often omit the influence of muscle strength on joint reaction forces, or consider simplify muscle system model, which results in strongly underestimated values. The implementation of advanced models of musculoskeletal system is possible due to the development of hardware and software. The usefulness of this method is confirmed by numerous papers such as (Nowakowska et al., 2017) which used this method in rehabilitation progress evaluation and (Guzik-Kopyto et al., 2016) which used this method for determination of loads in upper limb during daily living activities. There are also works on the use of

Andrzej Bieniek, MSc. Eng.: Department of Biomechatronics, Silesian University of Technology, ul. Roosevelta 40/114; 41800, Zabrze; PL, Andrzej.Bieniek@polsl.pl

** Assocciate Professor Robert Michnik, PhD., DSc. Eng.: Department of Biomechatronics, Silesian University of Technology, ul. Roosevelta 40/119; 41-800, Zabrze; PL, Robert.Michnik@polsl.pl

*** Piotr Wodarski, PhD. Eng.: Department of Biomechatronics, Silesian University of Technology, ul. Roosevelta 40/125; 41800, Zabrze; PL, Piotr.Wodarski@polsl.pl

$\dagger \quad$ Miłosz Chrzan, MSc. Eng.: Department of Biomechatronics, Silesian University of Technology, ul. Roosevelta 40/114; 41800, Zabrze; PL, Milosz.Chrzan@polsl.pl

* Jacek Jurkojć, PhD. Eng.: Department of Biomechatronics, Silesian University of Technology, ul. Roosevelta 40/125; 41800, Zabrze; PL, Jacek.Jurkojc@polsl.pl 
virtual reality in ergonomic research and rehabilitation (Jurkojć et al., 2016). Using virtual reality technology is possible to make custom biomechanical measurements and prototyping of ergonomic solutions, i.e. different workplace concepts, optimized to minimize loads of musculoskeletal system (Hu et al., 2011). The aim of the study was to determine the impact of considering a complex musculoskeletal system model on the values of musculoskeletal system loads during selected variants of stoop and squat positions of maintaining the object above the ground. The reaction forces in the L5S1 intervertebral disc were analyzed, which is justified by the literature. The reaction force in knee joint was also analyzed, this joint is also exposed to overload but is often overlooked in analyzes focusing on the spine loads. In addition, the value of the goal function, which was the sum of squares of muscle forces, was determined. This parameter corresponds to the energy expenditure.

\section{Methods}

To determine the loads in the musculoskeletal system, the AnyBody software and the whole-body model "standing model" provided with the software were used. This model is characterised by estimation of the ground reaction forces which allow to determine the skeletal muscle system loads for simulated conditions without biomechanical surveys. The model description and characteristic are available on the software manufacturer's website. This model has been positively verified many times, for example by (Rajaee et al., 2015). A total of 10 cases of body posture during maintenance of the object above the ground has been analysed, 5 in the stoop position and 5 in the squat position (Tab. 1).

Tab. 1: Analysed positions parameters.

\begin{tabular}{|c|c|c|c|c|c|c|c|c|c|c|c|}
\hline & \multirow[b]{2}{*}{ number of position } & \multicolumn{5}{|c|}{ stoop } & \multicolumn{5}{|c|}{ squat } \\
\hline & & 1 & 2 & 3 & 4 & 5 & 1 & 2 & 3 & 4 & 5 \\
\hline \multirow{4}{*}{$\begin{array}{l}\frac{0}{00} \\
\frac{0}{\Xi}\end{array}$} & knee [deg] & 0 & 10 & 10 & 10 & 10 & 130 & 130 & 130 & 130 & 130 \\
\hline & hip [deg] & 90 & 90 & 100 & 80 & 100 & 130 & 130 & 80 & 130 & 120 \\
\hline & lumbar spine [deg] & -50 & -50 & -30 & -50 & -20 & -10 & 0 & -70 & -20 & -40 \\
\hline & shoulder [deg] & 100 & 100 & 100 & 100 & 100 & 30 & 30 & 70 & 60 & 80 \\
\hline \multirow{3}{*}{ : } & $\operatorname{com}[\mathbf{m}]$ & 0 & 0.1 & 0 & 0.25 & 0.15 & 0.05 & 0.1 & 0 & 0.05 & 0.05 \\
\hline & hand saggital [m] & 0.07 & 0.15 & 0.24 & 0.30 & 0.39 & 0.07 & 0.15 & 0.24 & 0.31 & 0.40 \\
\hline & hand vertical [m] & 0.17 & 0.18 & 0.22 & 0.17 & 0.21 & 0.17 & 0.18 & 0.23 & 0.18 & 0.21 \\
\hline
\end{tabular}

The positions have been selected so that the hand positions relative to the ankle joint of corresponding cases are pairwise close together. The positions of the hand in the vertical axis is close to 0.2 meters. The following degrees of freedom of the model were used: shoulder flexion, lumbar spine flexion, hip flexion, knee flexion and ankle flexion, determined by modification of the centre of pressure (COP) position. Model profiles are presented in the table (Fig. 1:). These positions were obtained by generating a grid of end positions of hand for a grid of joint angles changed every 10 degrees and COP positions every centimetre and then randomly selecting models that met the assumptions. The remaining values were set as neutral joint positions. The load was set as $5 \mathrm{~kg}$ additional mass in each hand.

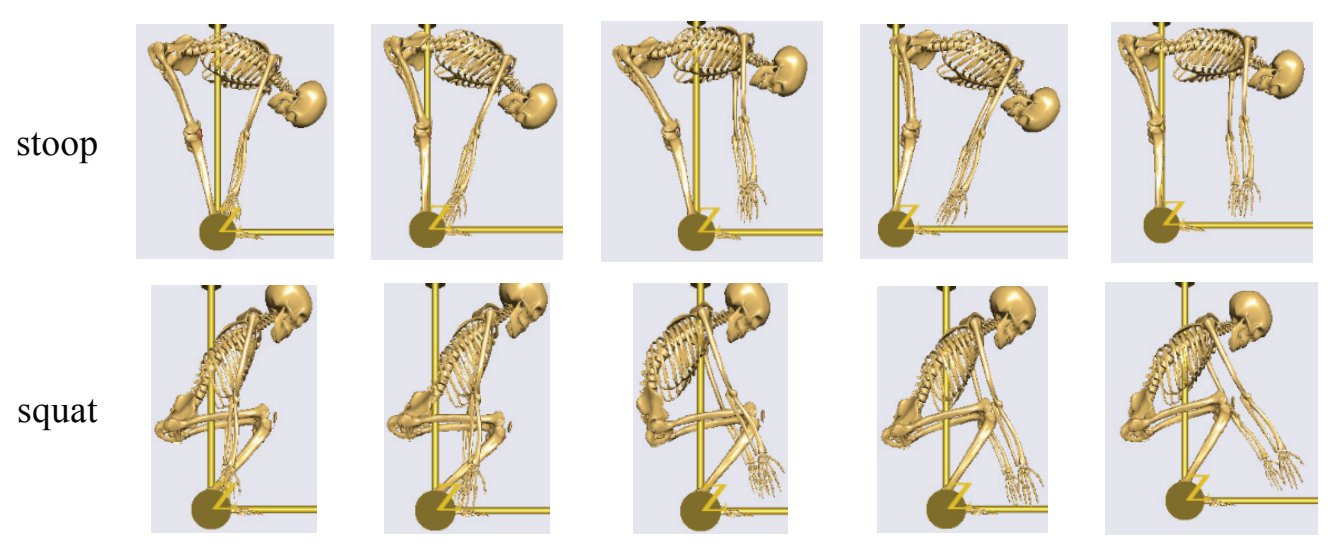

Fig. 1: Analysed positions profiles. 
An inverse dynamic analysis was performed, obtaining the values of the reaction forces and moments in the joints and the value of muscle force.

\section{Results}

Resultant reactions in the knee joint and disc between L5 and S1 vertebrae (Fig. 2) were analysed as well as the value of the goal function (Fig. 1), which was the sum of squares of muscle forces. The results are presented in diagrams as the dependence of the values on the distance of the hand from the ankle joint along the sagittal axis for stoop and squat positions and for the model considering the muscles and not taking them into account.

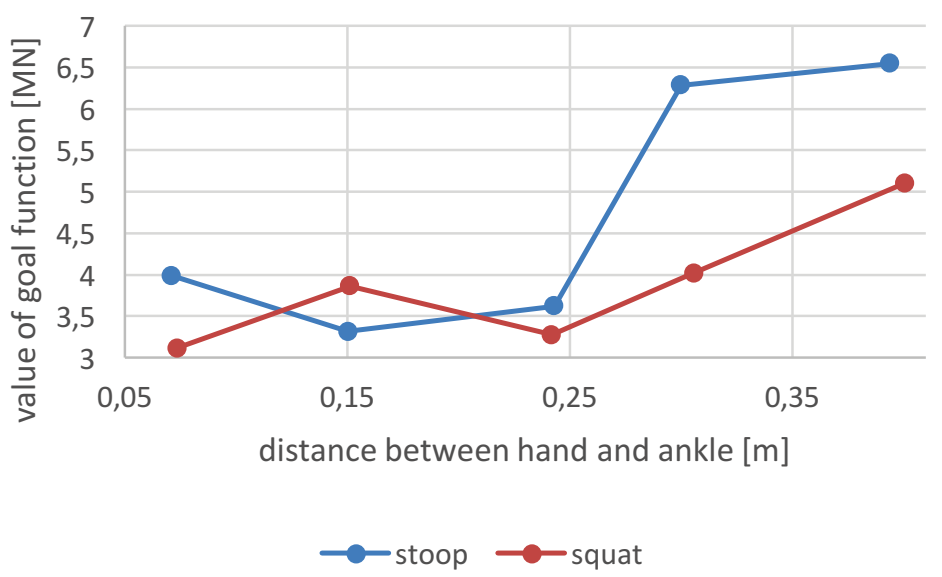

Fig. 2: Value of goal function.
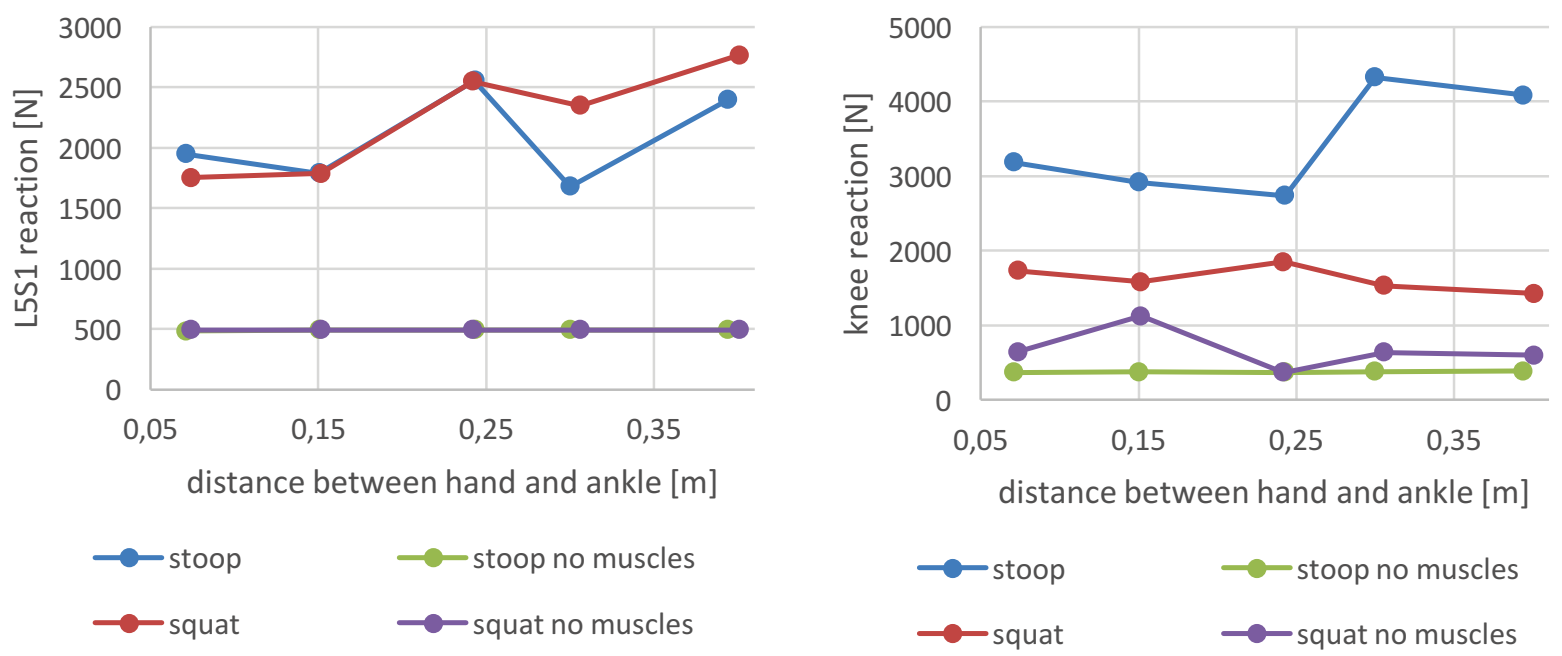

Fig. 3: Resultant joint reaction.

The results obtained for the L5S1 segment without the use of muscles have a constant value, because with the change of position only the distribution of forces for the axis changes without changing the resultant force. For hand positions closed to the position of the ankle one can notice similar values of the target function and reaction forces in the L5S1 intervertebral disc for both analysed techniques. For higher values of the distance of the hand from the ankle you can see higher values of muscle strength and lower values of reaction force in the intervertebral disc L5S1 for stoop position. One can notice a greater reaction force in the knee joint for the stoop position, which may be due to muscle action. 


\section{Conclusions}

It can be noticed that the values without considering the muscles are about an order of magnitude smaller, which indicates the necessity to use models that allow for the inclusion of muscle strength in the calculation of musculoskeletal system loads. One can see getting similar values to the found literature references, taking into account other study conditions (Rajaee et al., 2015; Dreischarf et al., 2016). However, there is no literature describing the operation of the entire muscle apparatus during lifting and hence general body fatigue caused by motor activities. The problem requires the calculation of a larger number of models, including in particular considering the influence of individual angles in the joints and taking into account the accelerations accompanying the lifting of objects.

\section{References}

Chang, C. C. et al. (2001) Biomechanical simulation of manual lifting using spacetime optimization, Journal of Biomechanics, 34(4), pp. 527-532. doi: 10.1016/S0021-9290(00)00222-0.

Van Dieën, J. H., Hoozemans, M. J. M. and Toussaint, H. M. (1999) Stoop or squat: A review of biomechanical studies on lifting technique, Clinical Biomechanics, pp. 685-696. doi: 10.1016/S0268-0033(99)00031-5.

Dreischarf, M. et al. (2016) In vivo loads on a vertebral body replacement during different lifting techniques, Journal of Biomechanics, 49(6), pp. 890-895. doi: 10.1016/j.jbiomech.2015.09.034.

Guzik-Kopyto, A. et al. (2016) Determination of Loads in the Joints of the Upper Limb During Activities of Daily Living, in Advances in Intelligent Systems and Computing, pp. 99-108. doi: 10.1007/978-3-319-39904-1_9.

$\mathrm{Hu}$, B. et al. (2011) Predicting real-world ergonomic measurements by simulation in a virtual environment, International Journal of Industrial Ergonomics. Elsevier, 41(1), pp. 64-71. doi: 10.1016/j.ergon.2010.10.001.

Jurkojć, J. et al. (2016) The Upper Limb Motion Deviation Index: A new comprehensive index of upper limb motion pathology, Acta of Bioengineering and Biomechanics, 19(2), pp. 175-185. doi: 10.5277/ABB-006982016-02.

Katsuhira, J. et al. (2013) Effect of Mental Processing on Low Back Load While Lifting an Object, Spine, 38(13), pp. E832-E839. doi: 10.1097/BRS.0b013e31829360e5.

Nowakowska, K. et al. (2017) Innovations in Biomedical Engineering, Innovation in biomedical engineering. Edited by M. Gzik et al. Cham: Springer International Publishing (Advances in Intelligent Systems and Computing). doi: 10.1007/978-3-319-47154-9.

Plamondon, A. et al. (2014) Lifting strategies of expert and novice workers during a repetitive palletizing task., Applied ergonomics, 45(3), pp. 471-81. doi: 10.1016/j.apergo.2013.06.008.

Rajaee, M. A. et al. (2015) Comparative evaluation of six quantitative lifting tools to estimate spine loads during static activities, Applied Ergonomics, 48, pp. 22-32. doi: 10.1016/j.apergo.2014.11.002.

Visser, S. et al. (2015) Lumbar compression forces while lifting and carrying with two and four workers, Applied Ergonomics, 50, pp. 56-61. doi: 10.1016/j.apergo.2015.02.007. 